\title{
Where do the conceptual models for behaviour change come from, and how are they used? A critical and constructive appraisal
}

\author{
Nathalie André ${ }^{\mathrm{a} \otimes}$ and Louis Laurencelle ${ }^{\mathrm{b}}$ \\ ${ }^{a}$ Centre de Recherches sur la Cognition et l'Apprentissage, Université de Poitiers \\ ${ }^{\mathrm{b}}$ Département des sciences de l'activité physique, Université du Québec à Trois-Rivières
}

\begin{abstract}
This essay aims to examine the nature of global conceptual models of human behaviour and their contribution to the advancement of scientific knowledge in the health and social sciences. Our perspective revisits the role that the researcher exerts in the face of theoretical models: should the researcher be an ambassador for a given model, or rather act as an independent examiner who challenges the model with his own ideas and methods, checks up its workings and strives to better understand behavioural processes? Is it the duty of the researcher to again exemplify the approximate descriptive pertinence of a well-known model? To the question: "Do the global or generalist conceptual models facilitate or rather jeopardize the understanding of data and scientific progress?", responses are given by focusing on the limitations of the methodologies intended to empirically validate global theoretical models and on the risk of ossifying the practice of research under the authority of consecrated paradigms. Alternative approaches are propounded to revalue strict ethological observation, introduce $a d$ hoc instead of global models and explore definite hypotheses in current research.
\end{abstract}

Keywords « Epistemology; Conceptual models; Validity; Predictive validity; Human ethology; Discovery.

Acting Editor $\square$ Denis Cousineau (Université d’Ottawa)

Reviewers

- Three anonymous reviewers

\section{Introduction}

Global conceptual models generally play the role of "developers" in scientific research endeavours, allowing us to give meaning to observable pieces of reality and think in a structured way about real life phenomena. They also serve as sources of inspiration for setting up new research and may prompt researchers to contrive new hypotheses. However, for decades of research on behaviour and behaviour change, conceptual ‘tought-up' models have stagnated, admitting but a few ephemeral variants, and if they are generally accepted as likely representations of reality, their success at predicting and controlling human behaviour is more than disappointing (e.g., Milne, Sheeran, \& Orbell, 2000; Webb \& Sheeran, 2006; Carpenter, 2010).

After putting forward a few definitions to set our vocabulary, we shall review current practices of researchers when using low predictive models or when their research results do not directly confirm the original stated model. These practices will be illustrated in the context of the models using the intention / behaviour relationship. Two main objections will be formulated around the prescriptive nature of the conceptualization of these models and the way confirmatory models are used to validate them. The status of global conceptual models will then be scrutinized in the light of a larger view of scientific research and development, conceptual models being contrasted with empirical, ethology-wise models and with experimental, laboratorywise ones, the big question being: Where do conceptual models for behaviour change come from?

\section{Definitions}

Since the main purpose of this essay is to discuss the use and misuse of global models, it is appropriate to define a 
few terms, albeit somewhat arbitrarily, as they pertain to the health and social sciences domains. We shall use the terms 'model', 'conceptual model' and 'global conceptual model' as mutually equivalent and referring to a structured sequential scheme that represents either a causal chain of determinants or a sequential chain of states in a given human behaviour.

These models belong to a set of high level scientific constructs that may be characterized as either empirical / descriptive, experimental / causal, or conceptual / prescriptive constructs which, for the sake of clarity, we shall designate with the terms 'portrait', 'theory', and 'model' respectively. Portraits are basically empirical and rely on systematic observation, as typified by the works of ethologists, whether in the animal or human realm. Laboratory and controlled experimentation, driven by cause - effect demonstrations, are the principal research modalities used for theory construction. Such theoretical models stem initially from pieces of previously acquired knowledge and pinpoint experimental tests, and they eventually lead to what may be called a theory, such as Underwood's theory of proactive interference on learning or Pavlov's classical conditioning theory. Conceptual models have another origin and a different function for research insofar as they are essentially 'thought up' constructs or schemata, based on human logic, common sense and simplified rules of consequence: their aim is to organize and give meaning to the complex phenomena of human behaviour. Such models furnish a template for interpreting and tentatively explaining observational data. Behaviour chain models such as Ajzen's Theory of planned behaviour (TPB), Rosenstock's Health belief model (HBM) and Rogers' Protection motivation theory (PMT) are well-known instances of this last category of scientific constructs.

Our stance concerns specifically models of this conceptual / prescriptive kind, as we challenge their heuristic value and their usefulness in studying behaviour change. Most of the phenomena under study in health sciences and humanities stand at a high level of complexity. Yet, the conceptual models used are often simplistic, research designs employed are framed and indeed adapted to the model instead of the specific hypothesis at stake. Moreover, the proof techniques used are aimed at 'confirming' the model rather than testing the data-specific hypothesis. These various aspects will be discussed in the following sections.

\section{Usual practices in Health Sciences when faced with low predictive models}

Global conceptual models in the contemporary scientific literature are privileged tools for interpreting and explaining the complex phenomena of society and human behaviour. They can also be used to inspire research by sug- gesting variables to be identified and hypotheses to test experimentally. Simultaneously testing the entire model will generate a whole series of data, some of them upholding some aspects of the model, others not: What can the researcher do in this rather familiar predicament?

When confronted with low predictive validity or replication failure, researchers in the domain of behaviour change have four options. First, they are generally prone to expand the chosen model trying to step up the predictive power of the model at hand with more or less success (e.g., Gaston \& Prapavessis, 2014; De Bruijn, Wiedemann, \& Rhodes, 2014). A second option is to make periodical updates of the model in line with the advancing theoretical debate (Schwarzer, 2014); however these modifications often lead to simplistic contrived solutions (or hypotheses), such as putting forth a "motivational coherence" factor (Sheeran \& Conner, 2017) defined by inter-attitudinal consistency, striving to strongly predict behaviour. Third, some other authors have evoked the possible replication of the original model's demonstration and its associated evidence with the aid of (simplifying) auxiliary assumptions, that is, supporting assumptions that link the theory to an actual observation (e.g., Trafimow \& Earp, 2016). These proposed approaches are not always suitable for behaviour-flow models because of the inherent irrationality of certain behaviours and their low reproducibility. Notwithstanding the said expedients, the above model's limitations and inadequacies are sufficient to explain its lack of predictive value in clinical research, insofar as patients often are asked to engage in problematic or idiosyncratic behaviours that interfere with habit and selfregulation (Rhodes \& De Bruijn, 2013; De Bruijn et al., 2014). Indeed, the behaviour to be predicted is generally a new or upgraded one, a changed behaviour, this new behaviour having to dislodge or supersede competing customary behaviours, bypass habits, remove barriers, accommodate to life context, all elements wherein lies the unpredicted and often overlooked part of behavioural variance.

A fourth option is to methodologically reconcile the initial, incomplete or ill-fitting model with the emerging model that would match their data. This upward reconciliation is fostered by the supporters of confirmatory statistical techniques (CFA, SEM) and is authorized by their leniency for imperfect models. The mathematical distance between observed data and the model's structure and variable is assessed by a Chi-squared loss function measure (Joreskog, 1969), wherefrom are derived various fit indices such as CFI, NNFI, TFI, SRMR, RMSEA. A significant Chisquared result indicates that the adjusted model overlooks some significant portion of the observed data variance, plainly a data-model mismatch. in such cases and under 
the confirmatory trend, and spurred by the available software, researchers then proceed to minute model-targeted corrections intended to improve the fit by cutting down the Chi-squared value, simultaneously upgrading the fix indices. The testing function of the Chi-squared is thus reverted to a gauging and adjusting role, through which the researcher updates and calibrates a new version of the model with the resulting fit statistics as a guide (see also Barrett, 2007; Steiger, 2007). Such a process is a twin to that of the traditional Least squares (or Chi-squared) fitting, a XIXth century principle and method used to fine-tune the parameters of a validated model, but not one for building or validating it. This approach leads to confirm the original 'but as amended' model to the detriment of actual observed differences, differences that might contain the real discoveries of a research: Does the modified model constitute an endorsement of the prototype model and, if not, how come does this initial model survive? The hindrance of global conceptual models appears at this level when all efforts are made to keep the preferred model, in spite of its observed discrepancies.

Research efforts in behaviour studies should review and even re-establish their descriptive rigor and thoroughness and implement models that bank on predictive validity. For instance, the validity of a global conceptual model is seldom, if ever, examined through the validity of its individual components, the psychometric soundness of its observed variables, or the appropriateness of statistical tools used. In fact, the present quasi-hegemony of the global conceptual model approach and its confirmatory modality are an invitation to the construction and testing of premature explanatory models. Specifically, our point is to heighten researchers' awareness of the use of observationbased predictive conceptual model, rather than prescriptive / descriptive models, the experimental evidence of which is quite problematic.

\section{The intention / behaviour relationship: an illustration}

In spite of many studies that have examined the role of intention in the prediction of behaviour in various domains such as politics, health, education, marketing, ecology and climate control, the models currently collapse when confronted with actual future outcomes (Head \& Noar, 2014; Sniehotta, Presseau, \& Araújo-Soares, 2014; Noar \& Zimmerman, 2005; McEachan, Conner, Taylor, \& Lawton, 2011).

Currently, theoretical behaviour-flow models in health and social psychology are based on the staging of relationships between variables that are rarely experimentally tested, i.e. independently validated, their predictive efficiency thus being impoverished. The customary and ofttimes use of these models in prediction and counseling is apt to jeopardize the status of the evidence and, consequently, the credence in scientific knowledge. To illustrate our stance, let us take an example resorting to the theory of reasoned action (Fishbein \& Ajzen, 1975). This model alleges that the immediate antecedent of behaviour is the person's intention to perform a task. At the origin of this proposal was citeaposd62 theory of propositional control that tested verbal conditioning and concept attainment in laboratory sessions (see Dulany, 1968). Dulany reported several tests of the model that support the need to include behavioural intention as a moderator between knowledge, beliefs, awareness and behaviour. However, Dulany was more interested in the process or set of mental computations made by the individual before an action and he examined the role of reinforcement on the choice of responses. In 1962, Dulany reported that "a theory that holds intention to precede all response explains too much, and, in an old-fashioned sense, is certainly false", adding that "individuals do not always do what they think they have to do, unless what they do are based on habits”. That notwithstanding, Fishbein's (1973) adaptation of these findings led to a nearly hegemonic use of intention, while no published experimental demonstration can be found that intention is first among all possible determinants of behaviour. Yet, current studies test the adequacy of the entire model, without ever testing seriously the relationship between intention and behaviour.

To illustrate, the intention-behaviour asymmetry in the health domain has been studied - not experimentally - by De Bruijn et al. (2014) by extending Ajzen's TPB (Ajzen, 1991) to 'action control theory and automaticity'. The authors have shown that the discordance between intention and behaviour is reduced when individuals report strong habits toward the target behaviour (in that study, fruit intake). This finding has been echoed in other health behaviours: e.g., Rhodes and De Bruijn (2013) in physical activity, and $\mathrm{Xu}, \mathrm{Li}$, and Zhanga (2013) by studying the intention to jaywalk by pedestrians. Another example is given by citing the experimental (and rare) study of Gaston and Prapavessis (2014), who efficiently combined protection motivation theory with action and coping planning from the health action process approach model (Schwarzer, 2008) to enhance physical activity during pregnancy. Nevertheless, it would be easier to list studies that have tested these global models in a mechanical manner, resorting only to run-of-the-mill confirmatory procedures (e.g., causal path analysis, CFA, SEM) with evidence that is, questionably, supported by descriptive fit indices (e.g., Dowd, Jung, Chen, \& Beauchamp, 2016, using protection motivation theory, Parschau et al., 2014, with the health action approach model). The confirmatory argument supported by such methods, arguably ambiguous in itself, is 
also regularly aided by doctoring the model, even tampering with it through a few structural adjustments. In this operation, the original observational context and its related information are grossly simplified if not altogether forgotten, as well as are the rationale and legitimate applicability of the helpful model corrections. In this context, the researcher's predominant effort comes down to simply match a changed model against the data. In these cases, more common than would be desirable, the recourse to confirmatory analysis is, consciously or not, chosen to serve the model, that is, to confirm it or, more simply, to illustrate it anew, rather than to represent and interpret the data. This is due, on the one hand, to the customary practice of setting up the study on the structure of the target model with all its variables and relationships, and, on the other hand, to the fact that the confirmatory modeling approach almost always confirms, and no truly competing model has been thought up or planned. Such untimely interventions, prompted blindly by the software for improving the data-model fit, would logically lead to a rejection of the model, whereas authors either fail to discuss them or they integrate these adjustments as ad hoc, 'innocuous' amendments. As they now stand, most global behaviour models are deemed valid in a prescriptive, somewhat dogmatic way, and generally "they make sense" and are intuitively if not empirically sound.

From a pragmatic standpoint, based on the previous observation, throwing away data that do not validate models could be looked at as a possible option, given the lax conditions of corroboration harbored by the methodologies used for model validation (e.g., Pashler \& Wagenmakers, 2012; Masicampo \& Lalande, 2012). Undoubtedly, quantifying a poorly defined or ill-measured phenomenon, emphasizing a particular causal factor during a short (or null, even retrospective) period and then calculating a predictive function for some criterion measure (occasionally contiguous to the predictive variable) can only produce precarious conclusions, not evidence. Thence, the researcher's mistrust of the probative value of his negative results, and his defensible option to discard data that do not conform to the model. However, this choice is scarcely based on those considerations, and generally, the conceptual, quasi-dogmatic legitimacy and support of the global model take precedence and clinch the question.

\section{What are the minimum conditions that make a model valid?}

Models are built for a specific purpose, and validation studies should allow them to fulfill it. The framework of the validation of a model is established according to predefined criteria measuring the achievement of this objective: the said criteria relate to the model's conceptual clarity, its the- oretical formulation and structure, and its methodological levers for verifiability (Bunge \& Ardila, 1987; Borsboom, Mellenbergh, \& van Heerden, 2004). Most scientific models comply with these conditions; however it is not sufficiently the case in the human behaviour modeling literature. Quantitative research methods should be subservient to the scientific method and, as such, should provide means for confronting theory against reality in order to reveal all that the theory does not encompass and do so in such a manner as to elicit and identify other explanations involving new lines of research. This process subsumes the use of conceptual and methodological handles for model invalidation. Replication failures, for instance, are customarily ascribed to an unsound experimental design, poor measurements, improper samples of participants, etc., while some of them point to the emergence and discovery of something new. Although the repeated confirmation of a model by means of acceptable fit indices may be worthwhile, a single rebuttal through a controlled experimental study may be needed to point out its overall inadequacy, with this invalidating research phase serving as an appropriate counterpart of, or even as a condition for the creative one (Bunge \& Ardila, 1987). Usually, the creative part cannot be ordered and hinges chiefly on someone's ingenuity and knowledge. As for the necessary and complementary pruning of obsolete or inadequate models, it is done by putting each of their definitional hypotheses through empirical tests and, when proven defective, by rejecting them. Confirmation of a model may be reassuring, even utilitarian, but as suggested by Bunge (2017), some destructive process must be engaged to flush out the imperfections and dross of old models, a mandatory step needed to bring to light their obsolescence and make space for a new and more satisfying one. Consider that the test and possible rejection of an inadequate hypothesis is a step forward, more so than is the umpteenth confirmation of a global model and its associated paraphernalia: indeed, as a statistician might say, "The null hypothesis is impossible to prove"!

Other demonstrations of validity (André, Loye, \& Laurencelle, 2015; Borsboom et al., 2004; Loye, 2018) could be outlined by making plain and explicative the link between what is concretely demonstrated by the validation procedure and the stated objective of the validation. In the same way as the validation of a psychometric test, the validation of a behaviour-flow model must account for the methodology used to prove its validity. Finally, the validity of a model or hypothesis, such as that of a psychometric test or of Ajzen's TPB, can be questioned in three respects: to prove / disprove the reality of the supposed relationships between variables and the conditions thereof, to define the limitations of the model, and to predict experimentally independent outcomes or consequences, foreseen in the 
model or not.

\section{Global conceptual model versus ad hoc model}

Models help to organize knowledge, formalize predictions, ensure consistency, deepen explanation, aid in the development of rigorous hypotheses and facilitate the cumulative development of knowledge ..., provided that they have been experimentally validated. Generating the evidence requires the existence of a model, yes, but it is possible to undertake a research based on an ad hoc model, a test-specific hypothesis, particularly when the validity of a possible globalizing model is into question. Such was the rule in the first few decades of experimental psychology, spurning global models. For the researcher, the practical issue is to decide whether an ad hoc model, essentially defined by and confined to the research hypothesis, should be inserted into some global conceptual model, in addition to conforming to recognized scientific facts. By waiving the global model or putting it at bay, the researcher who believes he is on the trail of something can develop a hypothesis for an original and specific causal mechanism and propose to make it experimental evidence, if possible by controlling at the same time for other sources of influence and explanation. Another researcher, equally brilliant and imaginative, may prefer to insert his or her research hypothesis within the framework of a global model: if the hypothesis is proven, it will then survive by itself, and the shelter of the global model may prevent it from being dismissed by the editorial reviewers; however, no substantive contribution has been added to the global model.

In sum, the current problem of health and social psychology research may lie at this level: researchers should (re-)learn criticism and reconsider model orthodoxy. Our standpoint is not behaviouristic (or Skinnerian!), one for which "data is data" and scientific enquiry should confine to data accumulation and sorting. It does not exclude the usefulness and contribution of theory or of psychosocial theorizing. However, it wants to reintroduce the inductive and probative approach in scientific reasoning, preferably to the prescriptive-illustrative trend of recent research practice. Health and social psychology could be inspired by physics (as such was the case in the early days of psychological research) or natural sciences, as their research endeavours are guided by observation, exploration, discernment and measurement, rather than being bridled by would-be theories. Subjective perceptions or intuitbased hypotheses should not be excluded from scientific practice: they should guide it, even if these perceptions will need to be reconstructed in an operative way to become an appropriate tool for scientific research. Research is conceived as a systematic and structured intellectual endeavour coveting knowledge either by identifying the variables accounting for a circumscribed process or by establishing concomitant or causal relationships among the variables associated with a phenomenon. The ultimate goal of this process is to propose an articulate, mechanistic (and causal), verifiable model integrating all its aspects, components, rules, a model also capable of predicting new, as yet unobserved outcomes. How are we to build knowledge in health and social psychology, and what is the place of the (strict) experimental method for research in these fields?

\section{Evidence in social and health psychology}

The aim of a theory is specifically to explain the mechanisms to which observed phenomena may be ascribed and to predict unexplored facts and new phenomena. For a theoretical model to have any claim for truth and practical usefulness, it is necessary that it be validated within an experimental methodology. It is under this condition that one can debate the ultimate legitimacy of a model and that the choice to reject or pursue it is sensible. Undoubtedly, some ecological contexts are not readily adapted for naturalistic observation and for experimentation, such as reallife situations, educational settings, social phenomena or clinical environments. Yet, as noted by de Waal (1989), an ethologist specializing on primates, harder research feats have been accomplished in natural settings such as studies on dolphins and arboreal monkeys! In any case, it is mandatory and ultimately fruitful to simplify the context and narrow down the evidence by focusing in turn on one or two relationships between variables and testing them experimentally (Head \& Noar, 2014).

Three methods of substantiating evidence in health and social psychology could be considered. First, given new and theoretically ambiguous data, one could search for a factor, a dimension or a model other than that proposed by the prevailing theory to account for the results obtained, based on other principles of explanation or on a different organization of the same ones. For instance, a study carried out by Arden and Armitage (2008) questioned the usefulness of the 'intention to implement' for explaining the progress from the preparation stage; the reason was that a gap had been identified between the preparation stage and subsequent stages which might be explained by volitional strategies such as implementation intentions. By using an experimental longitudinal design, the authors randomly assigned participants to the experimental (implementation intention) or control conditions. A discriminant function analysis indicated that implementation intentions spur people to move from contemplation to the preparation stage, adding substantive evidence and predictive variance to the pertinence of the transtheoretical model when combined with other models.

A second way of establishing credible scientific evi- 
dence would be to pinpoint an empirical phenomenon, a variable, a dimension of reality that is still unexplored but that the theory at stake should explain, predict or control, and to see whether the experiment bears it out. In a study published by Seibert, May, Fitzgerald, and Fincham (2016), the authors investigated the causal relationship between burnout and the self-regulatory strength model of selfcontrol (Baumeister, Bratslavsky, Muraven, \& Tice, 1998) in undergraduate students. They hypothesized and proved that the deleterious effects of school burnout on the performance task were stronger under lower levels of state self-control. The authors then surmised that the mediation effect of self-control on this burnout / performance relation would in turn be influenced by the depletion effect. Participants were randomly divided into a high or low condition of an experimental motivational depletion task. Individuals from the high depletion task experienced a lower state self-control, demonstrating at the same time a stronger negative association between burnout and arithmetic performance.

A third proposal would be to cogitate about the ramifications or remote consequences (or deductions) consistent with the theory at stake and formulate a prediction, then verify whether the prediction is some way or not. This proposal is distinct from the previous two insofar as it has its inception in the theoretical model itself and not in the reference or experimental domain. It stems from a generic continuation or extrapolation of the model, without drawing from any corresponding actual past realization. Underwood (1957) was intrigued by the forgetting of single items regularly observed in studies on verbal memorization (McGeoch, 1932) and its given interpretation. More particularly, after a learning task performed in the laboratory and retention being measured a week later, the forgetting regularly observed in these studies was solely attributed to retroactive interference, that is, the pervasive and decaying effects of daily life activities during the week. Underwood questioned this explanation, favouring instead a proactive inhibition factor, that is, the actively interfering effects brought about by similar previously learned material. The greater the number of word lists memorized, the more difficult it was to acquire a new one, and the more forgetting occurred, independently of the learning /recall interval.

\section{Global models: a help or a hindrance?}

To the question: "Do the global or conceptual models facilitate or undermine data understanding and scientific progress?", there are two alternative responses. On the one hand, when the new data obtained are consonant with the model parameters and confirm the model's predictions, data understanding is ensured while the model is upheld, and its scope may be widened. On the other hand, in case of model failures (low predictive efficiency, contravariant or orphan data, validated competing explanations, etc.), the researcher is prone to look at the problematic results at as the model's amendable imperfections and, in most publications, these are not pursued nor resolved, with the data being disparaged in favour of aprioristic and unproductive conceptions. This response to model's incompatible data is obviously inadequate and unscientific, and it might impede scientific progress. A simple, more focused conceptual model would better isolate and integrate the relevant components in a parsimonious manner, as it is tailored down to its validated components and allows for understandable, more precise, shared communication on the meaning of a phenomenon. Finally, what is the role of global conceptual models? The scientific study of any phenomenon may be described as consisting of three interlocked phases, observation/description, comprehension/explanation, prediction/control of the said phenomenon. Symmetrically to this process, we may recall our categorized theoretical models, i.e. descriptive/ethological, conceptual/prescriptive, experimental/causal, among which the place and role of conceptual modelling appears sound and necessary: it serves to bridge the large gap between observation and control, providing the ideas and speculative rationalization that will permit and spur experimentation. Indeed, the "conceptualization" of a phenomenon lies in the second phase of the scientific process, and it follows and must follow a systematic observational phase, one akin to the large-scale studies undertaken by animal ethologists. On this question, ethologist de Waal (1989), already mentioned, writes:

"To understand the human species is a particularly challenging task. Because a truly objective view of one's own kind is impossible to achieve, it is not surprising that so many schools of thought and so many conflicting theories exist. Even though there is room for all these viewpoints, one approach meets with general hostility for scientists specialized in human behaviour - the approach of the biologist. Yet it seems to me that if the biological perspective is so much at odds with all the others, there is all the more reason to consider it. It is not through ignoring divergent view that scientific progress is achieved.” (de Waal, 1989, p. 229-230)

De Waal deplores also the shortage of anthropological work done on humans, and that current models are based more on interpretive and moral judgments of behaviour than stemming from observation. Such statements could serve as a considered response to our introductive interro- 
gation: Where do the conceptual human behaviour models come from?

\section{References}

Ajzen, I. (1991). The theory of planned behavior. Organizational Behavior and Human Decision Processes, 50, 179-211.

André, N., Loye, N., \& Laurencelle, L. (2015). La validité psychométrique : Un regard global sur le concept centenaire, sa genèse, ses avatars. Mesure et Evaluation en Education, 27, 125-148.

Arden, M. A., \& Armitage, C. J. (2008). Predicting and explaining transtheoretical model stage transitions in relation to condom-carrying behavior. British Journal of Health psychology, 13, 719-735. doi:10.1348/ 135910707X249589

Barrett, P. (2007). Structural equation modelling: Adjudging model fit. Personality and Individual Differences, $42,815-824$.

Baumeister, R. F., Bratslavsky, E., Muraven, M., \& Tice, D. M. (1998). Ego depletion: Is the active self a limited resource? Journal of Personality and Social Psychology, 74, 1252-1265.

Borsboom, D., Mellenbergh, G. J., \& van Heerden, J. (2004). The concept of validity. Psychological Review, 111, 1061-1071. doi:10.1037/0033-295X.111.4.1061

Bunge, M. (2017). Evaluating scientific research projects: The units of science in the making. Foundations of Science, 3, 455-469. doi:10.1007/s10699-015-9474-3

Bunge, M., \& Ardila, R. (1987). The philosophy of psychology. New York: Springer.

Carpenter, C. J. (2010). A meta-analysis of the effectiveness of health belief model variables in predicting behavior. Health Communication, 25, 661-669. doi:10.1080/ 10410236.2010.521906

De Bruijn, G.-J., Wiedemann, A., \& Rhodes, R. E. (2014). An investigation into the relevance of action planning, theory of planned behavior concepts, and automaticity for fruit intake action control. British Journal of Health Psychology, 19, 652-669. doi:10 .1111/bjhp . 12067

de Waal, F. B. M. (1989). Behavioral contrasts between bonobo and chimpanzee. In L. A. M. Heltne (Ed.), $P$ (pp. 154-175). Understanding Chimpanzees, . Cambridge: Harvard University Press.

Dowd, A. J., Jung, M. E., Chen, M. Y., \& Beauchamp, M. R. (2016). Prediction of adherence to a gluten-free diet using protection motivation theory among adults with coeliac disease. Journal of Human Nutrition and Dietetic, 29, 391-398. doi:10.1111/jhn.12321
Dulany, D. E. (1962). The place of hypotheses and intentions: An analysis of verbal control in verbal conditioning. Journal of Personality, 30, 102-129.

Dulany, D. E. (1968). Awareness, rules, and propositional control: A confrontation with s-r behavior theory. In Dixon \& D. Horton (Eds.), $T$ (pp. 340-387). Verbal behavior: Prentice-Hall.

Fishbein, M. A. (1973). The prediction of behavior from attitudinal variables. In C. D. Mortensen \& K. K. Sereno (Eds.), Advances in communication research (pp. 331). New York: Harper \& Row.

Fishbein, M. A., \& Ajzen, I. (1975). Belief, attitude, intention and behavior: An introduction to theory and research. MA, Addison Wesley: Reading.

Gaston, A., \& Prapavessis, H. (2014). Using a combined protection motivation theory and health action process approach intervention to promote exercise during pregnancy. Journal of Behavioral Medicine, 37, 173184. doi:10.1007/s10865-012-9477-2

Head, K. J., \& Noar, S. M. (2014). Facilitating progress in health behavior theory development and modification: The reasoned action approach as a case study. Health Psychology Review, 8, 34-52. doi:10 . 1080 / 17437199.2013.778165

Joreskog, K. G. (1969). A general approach to confirmatory factor analysis. Psychometrika, 34, 183-202. doi:10 . 1007/BF02289343

Loye, N. (2018). Et si la validité était plus qu'une suite de procédures techniques? Mesure et Évaluation en Éducation, 41, 97-123.

Masicampo, E. J., \& Lalande, D. R. (2012). A peculiar prevalence of $\mathrm{p}$ values just below .05. The Quarterly Journal of Experimental Psychology, 65, 2271-2279. doi:10. 1080/17470218.2012.711335

McEachan, R. R. C., Conner, M., Taylor, N. J., \& Lawton, R. J. (2011). Prospective prediction of health-related behaviors with the theory of planned behavior: A metaanalysis. Health Psychology Review, 5, 97-144. doi:10. 1080/17437199.2010.521684

McGeoch, J. A. (1932). Forgetting and the law of disuse. Psychological Review, 39, 352-370.

Milne, S., Sheeran, P., \& Orbell, S. (2000). Prediction and intervention in health-related behavior: A metaanalytic review of protection motivation theory. Journal of Applied Psychology, 30, 106-143.

Noar, S. M., \& Zimmerman, R. S. (2005). Health behavior theory and cumulative knowledge regarding health behaviors: Are we moving in the right direction? Health Education Research, 20, 275-290. doi:10.1093/ her/cyg113

Parschau, L., Barz, M., Richert, J., Knoll, N., Lippke, S., \& Schwarzer, R. (2014). Physical activity among adults 
with obesity: Testing the health action process approach. Rehabilitation Psychology, 59, 42-49. doi:10. 1037/a0035290

Pashler, H., \& Wagenmakers, E.-J. (2012). Editors' introduction to the special section on replicability in psychological science: A crisis of confidence? Perspectives on Psychological Science, 7, 528-530. doi:10 . 1177 / 1745691612465253

Rhodes, R. E., \& De Bruijn, G. J. (2013). How big is the physical activity intention-behavior gap? A metaanalysis using the action control framework, 18, 296309. doi:10.1080/17437199.2013.814921

Schwarzer, R. (2008). Modeling health behavior change: How to predict and modify the adoption and maintenance of health behaviors. Applied Psychology, 57, $1-29$.

Schwarzer, R. (2014). Life and death of health behavior theories. Health Psychology Review, 8, 53-56. doi:10.1080/ 17437199.2013.810959

Seibert, G. S., May, R. W., Fitzgerald, M. C., \& Fincham, F. D. (2016). Understanding school burnout: Does selfcontrol matter? Learning and Individual Differences, 49, 120-127. doi:10.1016/j.lindif.2016.05.024

Sheeran, P., \& Conner, M. (2017). Improving the translation of intentions into health actions: The role of motiva- tional coherence. Health Psychology, 36, 1-11. doi:10. 1037/hea0000553

Sniehotta, F. F., Presseau, J., \& Araújo-Soares, V. (2014). Time to retire the theory of planned behavior. Health Psychology Review, 8, 1-7. doi:10.1080/17437199.2013. 869710

Steiger, J. H. (2007). Understanding the limitations of global fit assessment in structural equation modelling. Personality and Individual Differences, 42, 893-898.

Trafimow, D., \& Earp, B. D. (2016). Badly specified theories are not responsible for the replication crisis in social psychology: Comment on klein. Theory and Psychology, 26, 540-548. doi:10.1177/0959354316637136

Underwood, B. J. (1957). Interference and forgetting. Psychological Review, 64, 49-60.

Webb, T. L., \& Sheeran, P. (2006). Does changing behavioral intentions engender behavior change? a metaanalysis of the experimental evidence. Psychological Bulletin, 132, 249-268.

Xu, Y., Li, Y., \& Zhanga, F. (2013). Pedestrians' intention to jaywalk: Automatic or planned? a study based on a dual-process model in china. Accident Analysis and Prevention, 50, 811-819. doi:10.1016/j.aap.2012.07.007

\section{Citation}

André, N., \& Laurencelle, L. (2020). Where do the conceptual models for behaviour change come from, and how are they used? a critical and constructive appraisal. The Quantitative Methods for Psychology, 16(1), 1-8. doi:10.20982/tomp. 16.1.p001 